\title{
Primary care access to computed tomography for chronic headache
}

\author{
Ralph Thomas, Alan Cook, Gavin Main, Tom Taylor, \\ Elizabeth Galizia Caruana and Robert Swingler
}

\author{
ABSTRACT \\ Background \\ The diagnostic yield of neuroimaging in chronic \\ headache is low, but can reduce the use of health \\ services.
}

Aim

To determine whether primary care access to brain computed tomography (CT) referral for chronic headache reduces referral to secondary care.

Design of study

Prospective observational analysis of GP referrals to an open access $\mathrm{CT}$ brain scanning service.

Setting

Primary care, and outpatient radiology and neurology departments.

\section{Method}

GPs in Tayside and North East Fife, Scotland were given access to brain CT for patients with chronic headache. All referrals were analysed prospectively over 1 year, and questionnaires were sent to referrers to establish whether imaging had resulted in or stopped a referral to secondary care. The Tayside outpatient clinic database identified scanned patients referred to the neurology clinic for headache from the start of the study period to at least 1 year after their scan.

\section{Results}

There were 232 referrals (55.1/100 000/year, 95\% confidence interval $=50.4$ to 59.9 ) from GPs in 59 $(82 \%)$ of 72 primary care practices. CT was performed on 215 patients. Significant abnormalities were noted in $3(1.4 \%)$ patients; there were $22(10.2 \%)$ nonsignificant findings, and 190 (88.4\%) normal scans. Questionnaires of the referring GPs reported that 167 $(88 \%)$ scans stopped a referral to secondary care. GPs referred $30(14 \%)$ scanned patients to a neurologist because of headache. It is estimated that imaging reduced referrals to secondary care by $86 \%$ in the follow-up period.

\section{Conclusion}

An open access brain CT service for patients with chronic headache was used by most GP practices in Tayside, and reduced the number of referrals to secondary care.

\section{Keywords}

headache disorders; computed tomography; open access; primary health care.

\section{INTRODUCTION}

Headache is one of the most common symptoms encountered in general practice, with an annual consultation rate of 4.44 per 100 registered patients. ${ }^{1}$ It is the single most common cause for neurology referral in the $\mathrm{UK},{ }^{2}$ accounting for up to $22 \%$ of GP referrals to neurologists. ${ }^{3}$ Chronic tension-type headache and migraine are the two most common headache types encountered in the UK population, both in primary care ${ }^{4}$ and specialist clinics. ${ }^{5}$ It has been argued that most primary headache can be managed in primary care, and that investigations are rarely needed. ${ }^{6}$ However, healthcare professionals often find the diagnosis and classification of headache difficult, ${ }^{7}$ and both healthcare professionals and patients worry about serious causes such as brain tumours. ${ }^{8}$

The role of imaging in this situation has been extensively reviewed, and guidance has been published for risk which stratifies patients according to presentation. ${ }^{9,10}$ Although pathology may be excluded by neuroimaging, the diagnostic yield is low, ${ }^{9,11,12}$ and the duration of reassurance for patients is short. ${ }^{13}$ Moreoever, there is a risk of finding coincidental abnormalities that may generate more anxiety. ${ }^{14}$ So, the US Headache

R Thomas, MRCP, clinical research fellow, NHS Lothian, Division of Clinical Neurosciences, Western General Hospital, Edinburgh. A Cook, FRCR, consultant radiologist; G Main, FRCR, consultant radiologist; T Taylor, FRCR, consultant radiologist, Department of Radiology; E Galizia Caruana, $M R C P$, neurology registrar; $\boldsymbol{R}$ Swingler, FRCP, consultant neurologist, Department of Neurology, NHS Tayside, Ninewells Hospital, Dundee.

\section{Address for correspondence}

Ralph GR Thomas, Division of Clinical Neurosciences,

Bramwell Dott Building, Western General Hospital,

Crewe Road South, Edinburgh EH4 2XU.

E-mail: ralph.thomas@nhs.net

Submitted: 26 August 2009; Editor's response: 3 November 2009; final acceptance: 5 January 2010.

(c)British Journal of General Practice. 2010; 60: 426-430.

DOI: 10.3399/bjgp10X502146 
Consortium Guidelines ${ }^{6}$ and the Scottish Intercollegiate Guidelines Network ${ }^{8}$ do not recommend neuroimaging for chronic headache in the absence of focal neurological signs. Nevertheless, observational studies indicate that brain scans are widely carried out on patients with normal neurological examinations. ${ }^{15-18}$

In a randomised trial to examine the effect of scanning of patients with chronic daily headache, referral rates to the neurology service were reduced from $17 / 74(23 \%)$ in the control group to $1 / 76$ $(1.3 \%)$ in the scanned group..$^{13}$ Although this study was restricted to selected patients referred to a trial, it raises the possibility that provision of an open access service might reduce chronic headache referral rates to secondary care for the entire population. Open access services have been shown to influence the management of patients in primary care by helping to avoid referrals to secondary care, ensuring referral to the appropriate specialty, and speeding up the time to diagnosis with a consultant opinion. ${ }^{19-21}$

The aim of this study was to estimate both the rate of referral of patients with chronic headache to an open access CT service, and the effect on referral rates to the neurology service in a defined population.

\section{METHOD}

The study was performed in Tayside and North East Fife, Scotland. The adult population of $421207^{22}$ is cared for by 309 GPs in 72 GP practices, and four full-time consultant neurologists, working in the local university teaching hospital, with outreach clinics in two district hospitals. Care is free at the point of access and few patients seek care outside the area.

The number of consultations for headache was estimated by applying the UK rate ${ }^{1}$ to the Tayside and North East Fife population, and the scan referral rate was the number of scan referrals as a proportion of this estimate.

All of the GPs were given access to refer patients directly to brain CT at three sites in the study area: Ninewells Hospital, Dundee; Perth Royal Infirmary, Perth; and Stracathro Hospital, Angus. Between these hospitals, approximately 7000 CT brain scans are carried out routinely each year. The referral protocol stated that patients should be aged 18 years or more, with a normal neurological examination, and symptoms consistent with chronic, unchanging headache, or of a non-acute nature such that secondary care referral for specialist opinion was not deemed necessary. The duration of headache was not specified.

All referrals were analysed prospectively for a 12-

\section{How this fits in}

In the absence of focal neurological signs, neuroimaging for chronic headache

is not recommended by US and Scottish Intercollegiate Guidelines Network

guidelines, but has been shown to reduce subsequent use of health services,

especially in patients with high levels of morbidity. In this study, an open access

brain CT service for patients with chronic headache was used by the majority of

GPs, and reduced referrals to secondary care.

month period from 1 April 2005 to 31 March 2006, and data were collected on the referral and referring practitioner and radiological findings. A follow-up questionnaire was sent to the referring GP with the CT scan report to establish whether the CT service had resulted in or stopped a referral to neurologists. The Tayside outpatient clinic database was used to determine which of the scanned patients were referred to a neurology clinic between 1 April 2005 and 31 March 2007, giving a follow-up period of at least 1 year after each patient's scan. GP referral letters and hospital records were then assessed, so that only those patients who were referred because of headache were included.

\section{RESULTS}

Between 1 April 2005 and 31 March 2006 the GPs in the study area referred 232 patients for brain CT (55.1/100 000/year, 95\% confidence interval $[\mathrm{Cl}]=$ 50.4 to 59.9). There were an estimated 18702 headache consultations, and the referral rate to the open access service was $1.24 \%$. A scan was not performed for $17(7.3 \%)$ of the patients because six appointments were cancelled, five patients did not attend, two request forms were incomplete, and four requests were declined because the radiologist did not think that the referrals complied with study criteria (one for memory loss, one for personality change, one for suspected stroke, and one for suspected transient ischaemic attack). So 215 $(92.7 \%)$ of the referred patients were scanned (51.0/100 000/year, $95 \% \mathrm{Cl}=46.3$ to 55.8 ), and two patients were recalled for second scans. Table 1 gives details of the age and sex of the 215 scanned

Table 1. Patients grouped according to whether or not they were referred to a neurology clinic for chronic headache.

\begin{tabular}{lccc} 
& $\begin{array}{c}\text { Referred to } \\
\text { neurology clinic }\end{array}$ & $\begin{array}{c}\text { Not referred to } \\
\text { neurology clinic }\end{array}$ & Total \\
\hline Male, $n$ & 14 & 80 & 94 \\
mean age in years (SD) & $47(16)$ & $48(15)$ & $48(15)$ \\
\hline Female, $n$ & 16 & 105 & 121 \\
mean age in years (SD) & $41(14)$ & $47(18)$ & $47(18)$ \\
\hline Total, $n$ & 30 & 185 & 215 \\
mean age in years (SD) & $44(15)$ & $48(17)$ & $47(17)$ \\
\hline
\end{tabular}


Table 2. Diagnoses in patients referred to neurologists.

\begin{tabular}{lc} 
Diagnosis & $n$ \\
\hline Tension-type headache & $6^{\mathrm{a}}$ \\
Tension-type headache and migraine & $6^{\mathrm{a}}$ \\
Migraine & 4 \\
Chronic daily headache not otherwise specified & 3 \\
Post-traumatic & 2 \\
Atypical facial pain & 1 \\
Trigeminal neuralgia & 1 \\
Thunderclap headache & 1 \\
Temporomandibular joint dysfunction & 1 \\
Not headache & 3 \\
Did not attend & 2 \\
\hline Total & 30 \\
\hline
\end{tabular}

alncludes one medication overuse headache.

patients, grouped according to whether or not they were referred to neurology clinic for chronic headache. The age range of the study population was $20-85$ years.

The CT scans of 215 patients identified three lesions which, in the opinion of the study neurologists, might have caused chronic headache. One patient had metastatic lung cancer, another patient had a meningioma, and a third patient had an arteriovenous malformation. It was concluded that 22 patients had non-significant findings including old infarcts $(n=7)$, other ischaemic changes $(n=7)$, arachnoid cysts $(n=2)$, sinusitis ( $n$ $=2)$, pineal cyst $(n=1)$, prominent deep cerebral vein $(n=1)$, probable artefact $(n=1)$, and other unspecified changes $(n=1)$, giving a $1.4 \%$ yield for significant pathological findings and $10.2 \%$ for nonsignificant findings; 190 (88.4\%) CT scans were reported to be normal.

The open access service was used by 140 (45\%) of 309 Tayside GPs from 59 (82\%) of the 72 GP practices. The highest number of CT requests from a single GP was seven: most GPs referred one patient. Completed questionnaires were obtained from 189 (88\%) of the referring GPs, and the

Table 3. Computed tomography results in patients referred to neurologists.

\begin{tabular}{lc} 
Findings & $n$ \\
\hline Normal & 17 \\
Dural/falcine calcification & 2 \\
Arachnoid/pineal cysts & 2 \\
Old lacunar infarct & 1 \\
Mild asymmetry & 1 \\
Small vessel disease & 1 \\
Incidental meningioma & 1 \\
\hline Total & $25^{a}$ \\
\hline
\end{tabular}

'Excludes two 'did not attend' and three 'without headache'. responders reported that, in 167 (88\%) of 189 cases with completed questionnaires, the scan had stopped a referral to secondary care; in $10(5 \%)$ cases the scan had caused a referral, and in 12 $(6 \%)$ cases a referral had been made to neurology service at the same time as the scan request.

There were 288.9 patient years of follow-up in 215 patients, giving a mean follow-up period of 1.3 years per patient (standard deviation = 0.29 years). Assessment of the Tayside outpatient clinic log and GP referral letters showed that, of the 215 patients scanned in 1 year, 30 (14\%) were referred to neurology clinics because of headache. Of these 30 patients, 12 (40\%) were referred before, or at the same time as their CT scan request, and the remaining 18 (60\%) were referred after their brain CT. The overall referral rate per 100 patient years was $10.4(95 \% \mathrm{Cl}=6.3$ to 14.5$)$. The diagnoses made by neurologists of the 30 patients referred to clinic are shown in Table 2, and the brain CT findings are shown in Table 3.

Five $(2.3 \%)$ patients died in the follow-up period: one patient had meningeal deposits of metastatic lung cancer seen on the study brain $\mathrm{CT}$; three other patients died of unrelated conditions including pneumonia $(n=1)$, pulmonary embolism $(n=1)$, and acute renal failure $(n=1)$. No information was available for one of the deceased patients.

\section{DISCUSSION}

\section{Summary of main findings}

The majority of GP practices in the study area used the open access service, and it is estimated that $1.24 \%$ of consultations for headache led to a referral to the open access service. Open access CT scans accounted for about $4 \%$ of the annual number of CT brain scans across Tayside. The yield of significant pathological findings in this study was $1.4 \%$, and this is consistent with findings in other populations of patients with chronic headache..$^{6,12,23}$

Results of the questionnaire sent to GPs who had used the service show that it may have prevented a neurology referral in $88 \%$ of cases. As the CT scan was said to have caused a referral in $5 \%$ of cases, one could assume that the predicted reduction in clinic referrals would be $83 \%$. This finding seems to have been borne out in the follow-up study, which showed that only $14 \%$ of the scanned patients were referred to neurology because of headache in the mean 1.3 years of follow-up per patient. Hypothetically, CT prevented a referral in $86 \%$ of cases. The $14 \%$ referral rate in scanned patients is higher than the rate observed in other much larger groups of patients with chronic headache., ${ }^{1,13}$ This may result from referral filter bias, as presumably these patients were referred because the GP was 
not certain of the diagnosis, or required advice about management. This is supported by the observation that in $12(6 \%)$ cases, the GPs referred their patients to neurologists at the same time as requesting CT. The neurologists' diagnoses of tension-type headache, migraine, or chronic daily headache in 19 out of 30 cases reflects the prevalence of these headache types in the general population. ${ }^{4}$

Is scanning a patient's brain for reassurance sufficient to render referral to secondary care unnecessary? Some patients will find reassurance in being given a diagnosis or an explanation of the problem, ${ }^{24}$ whether this comes from a primary or secondary care provider, and others will not. However, it could be argued that the GP is best placed to identify those patients most likely to be reassured by a negative or normal scan, and the present study indicates that the majority of GPs were able to manage scanned patients in primary care during the follow-up period.

\section{Strengths and limitations of the study}

This prospective observational study puts into a population-based context the findings of Howard et al, who showed that patients with chronic daily headache who were randomised to brain scanning had lower rates of referral to neurological services. ${ }^{13}$ First, it shows the likely level of uptake of the service is quite low, at $1 \%$ of consultations. Secondly, a questionnaire sent to referring GPs was used to estimate that a referral to the neurology service was avoided for $88 \%$ of scanned patients during the study period. Thirdly, it was possible to measure actual referral rates in the follow-up period to confirm this.

The observational study design has some limitations. First, because one of the study aims was to observe the effect of CT on usual clinical practice, GPs were not asked to classify the type or chronicity of headaches using the International Classification of Headache Disorders. ${ }^{25}$ Instead, the GPs were allowed to scan patients within the bounds of the referral protocol. Second, it was not possible to evaluate the GPs' management of patients with headache, before and after the scans, or to compare it to specialist care. This would require a randomised trial comparing GP and specialist practice, with and without access to imaging. Third, long-term studies have not been carried out to look for trends in rates of referral to the open access CT service. Nor did the study look at the effect on referrals to non-neurology clinics, such as general medicine, ophthalmology, and otorhinolaryngology. Lastly, it is important to consider the identification of significant coincidental findings, together with the small risk associated with exposure to radiation. There may also have been benefits of the open access service if its availability changed the management of people with headache who were not scanned.

\section{Comparison with existing literature}

Howard et al carried out a randomised controlled trial of patients with chronic daily headache in which the intervention was the offer of a brain scan. ${ }^{13}$ They found that neuroimaging reassured patients about the cause of their headache when they were reassessed at 3 months, but this effect was not sustained at 1 year. Nevertheless, this study did demonstrate that scanned patients had a lower rate of referral to the neurology service. However, the study was a trial of selected patients with chronic daily headache. It did not provide an estimate of the likely referral rates for patients with chronic daily headache, nor did it show an actual effect of reduced referral rates at a population level. The present study allowed an estimate that $1 \%$ of consultations led to a referral for CT, and that referrals of scanned patients to the neurology service were reduced by $86 \%$.

\section{Implications for clinical practice}

The primary reason for arranging neuroimaging must remain its clinical indication, but the results of this study suggest that open access scanning may give GPs more confidence in managing the small minority of patients for whom a normal scan will provide reassurance and prevent secondary referral.

\section{Competing interests}

The authors have stated that there are none.

\section{Acknowledgements}

The authors wish to thank Wendy Mutch for administrative assistance.

\section{Discuss this article}

Contribute and read comments about this article on the Discussion Forum: http://www.rcgp.org.uk/bjgp-discuss

\section{REFERENCES}

1. Latinovic R, Gulliford M, Ridsdale L. Headache and migraine in primary care: consultation, prescription and referral rates in a large population. J Neurol Neurosurg Psychiatry 2006; 77(3): 385-387.

2. Goadsby PJ, Boes C. Chronic daily headache. J Neurol Neurosurg Psychiatry 2002; 72 Suppl 2: ii2-ii5.

3. Bekkelund SI, Albretsen C. Evaluation of referrals from general practice to a neurology department. Fam Pract 2002; 19(3): 287-289.

4. Rasmussen BK. Epidemology of headache. Cephalalgia 2001; 21(7): 774-777.

5. Dowson AJ. Analysis of the patients attending a specialist UK headache clinic over a 3-year period. Headache 2003; 43(1): 14-18.

6. Frishberg $\mathrm{BM}$, Rosenberg JH, Matchar $\mathrm{DB}$, et al. US Headache Consortium. Evidence-based guidelines in the primary care setting: neuroimaging in patients with non-acute headache. The American Academy of Neurology.

https://www.aan.com/professionals/practice/pdfs/gl0088.pdf (accessed 13 Jan 2010). 
7. Kernick D, Stapley S, Hamilton W. GPs' classification of headache: is primary headache underdiagnosed? Br J Gen Pract 2008; 58(547): $102-104$

8. Scottish Intercollegiate Guidelines Network. Diagnosis and management of headache in adults. Guideline 107. Edinburgh: SIGN, 2008. http://www.sign.ac.uk/pdf/sign107.pdf (accessed 13 Jan 2010).

9. Sobri M, Lamont AC, Alias NA, Win MN. Red flags in patients presenting with headache: clinical indications for neuroimaging. $\mathrm{Br} \mathrm{J}$ Radiol 2003; 76(908): 532-535.

10. Kernick DP, Ahmed F, Bahra A, et al. Imaging patients with suspected brain tumour: guidance for primary care. Br J Gen Pract 2008; 58(557): 880-885.

11. Benamore RE, Wright D, Britton I. Is primary care access to CT brain examinations effective? Clin Radiol 2005; 60(10): 1083-1089.

12. Sempere AP, Porta-Etessam J, Medrano V, et al. Neuroimaging in the evaluation of patients with non-acute headache. Cephalagia 2005; 25(1): $30-35$.

13. Howard L, Wessely S, Leese M, et al. Are investigations anxiolytic or anxiogenic? A randomised controlled trial of neuroimaging to provide reassurance in chronic daily headache. J Neurol Neurosurg Psychiatry 2005; 76(11): 1558-1564

14. Hayward R. VOMIT (victims of modern imaging technology) — an acronym for our times. BMJ 2003; 326: 1273

15. Evans RW. Diagnostic testing for the evaluation of headaches. Neurol Clin 1996; 14(1): 1-26.
16. Lewis DW, Dorbad D. The utility of neuroimaging in the evaluation of children with migraine or chronic daily headache who have normal neurological examinations. Headache 2000; 40(8): 629-632.

17. Wang HZ, Simonson TM, Greco WR, Yuh WT. Brain MR imaging in the evaluation of chronic headache in patients without other neurologic symptoms. Acad Radiol 2001; 8(5): 405-408.

18. Tsushima Y, Endo K. MR imaging in the evaluation of chronic or recurrent headache. Radiology 2005; 235(2): 575-579.

19. White PM, Halliday-Pegg JC, Collie DA. Open access neuroimaging for general practice: diagnostic yield and influence on patient management. Br J Gen Pract 2002; 52(474): 33-35.

20. Apthorp LA, Daly CA, Morrison ID. Direct access MRI for genera practitioners: influence on patient management. Clin Radiol 1998; 53(1): 58-60

21. Stoddart PGP, Hall SG. Radiology is valuable to GPs; but who pays? Clin Radiol 1989; 40(2): 183-185.

22. United Kingdom Census, 2001, Scottish Neighbourhood Statistics. http://www.sns.gov.uk (accessed 13 Jan 2010).

23. Dumas MD, Warwick Pexman JH, Kreeft JH. Computed tomography evaluation of patients with chronic headache. CMAJ 1994; 151(10): $1447-1452$.

24. Goadsby PJ. To scan or not to scan in headache? BMJ 2004 329(7464): 469-470.

25. IHS Classification (IHCD-II). 2nd edn. http://ihsclassification.org/en/ (accessed 13 Jan 2010). 